\title{
Prostatic Ductal Adenocarcinoma: An Unusual Subtype of Prostate Cancer
}

\author{
Dinesh Kumar, MBBS, MS* and John Lobo, MD \\ Urology Surgeons, PC, Grand Rapids, Michigan, USA
}

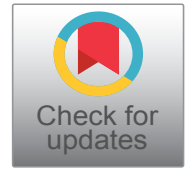

*Corresponding author: Dinesh Kumar, MBBS, MS, Urology Surgeons, PC, Grand Rapids, Michigan, USA

\begin{abstract}
Ductal adenocarcinoma of the prostate (DAP) is defined as malignant epithelial cells filling large prostatic acini ducts, with preservation of basal cells forming either solid or dense cribriform patterns or a loose cribriform or micropapillary pattern with either marked nuclear atypia (nuclear size six times normal or larger) or Nonfocal comedonecrosis. DAP is a rare variant of prostate cancer, and has poorlyidentified clinical characteristics. DAP is characterized by a high volume and high-grade disease, with an aggressive behavior. Few cases have been previously reported in Asian males and DAP is more commonly reported in Caucasian males over 70 years of age. In the present study, a 87-yearold Asian American male patient that demonstrated nodular prostate with lower urinary tract symptoms and elevated prostatic specific antigen levels underwent a prostate biopsy. The patient was subsequently diagnosed with DAP. He was successfully managed with combined Radiation therapy and Androgen deprivation therapy. During the 1 -year follow up, the patient remained progression free. A review of the literature was conducted in order to assess the clinical course, diagnosis, prognosis and optimal management for DAP. The review indicated that DAP is more likely to demonstrate an aggressive clinical course and an unfavorable prognosis; therefore, once the diagnosis has been confirmed, an aggressive management strategy is recommended for the patient, even in the case of metastatic disease.
\end{abstract}

\section{Keywords}

Nuclear atypia, Radiation therapy, Androgen deprivation therapy, Prostatic specific antigen

\section{Introduction}

Malicow and Pachter first described a case of PDA in 1967 and were of the opinion that the tumor had "endometrial features" suggesting its origin from the prostatic utricle (Uterus masculinus) [1]. However, sub- sequent studies have clearly confirmed the prostatic origin of DAP, which is supported by a favorable response to orchiectomy, the expression of prostatic-specific antigen (PSA) and ultrastructural findings [2].

DAP is a rare subtype of prostate cancer (PCa) that has a poorly-understood etiology, and more commonly occurs in Caucasian males that are $>70$ years of age [3]. It usually occurs in the prostatic urethra around the verumontanum, and its prognosis is generally thought to be worse than for acinar adenocarcinoma [4]. A previous study that reviewed the histological slides of 2,600 prostatic carcinomas reported a prevalence of $0.4-0.8 \%$ for pure ductal adenocarcinoma and $5 \%$ for mixed ductal adenocarcinoma [5]. Here we present a case report and review the relevant literature concerning DAP in order to summarize the clinical features, histological characteristics and therapeutic strategies for DAP.

\section{Case Report}

87-year-old Asian American male with past history of nodular enlarge prostate with lower urinary tract symptoms (well managed with Alfuzosin $10 \mathrm{mg}$ PO daily) and long-standing history of elevated and fluctuating PSA up to $16.5 \mathrm{ng} / \mathrm{ml}$. He was evaluated with $\mathrm{CT}$ abdomen and pelvis for persistent RLQ and bilateral groin pain in Nov 2016 and found to have enlarge right pelvic lymph node concerning for lymph node metastasis and lobular enlarge prostate concerning for neoplasm. Lymph node biopsy was not possible due to location. Therefore, a transrectal prostate biopsy was performed and the specimens revealed high grade prostate carcinoma, Gleason 9 and 8, with ductal differentiation and highgrade PIN. A bone scan and chest computed tomography (CT) scans indicated no metastases. Due to the long-life

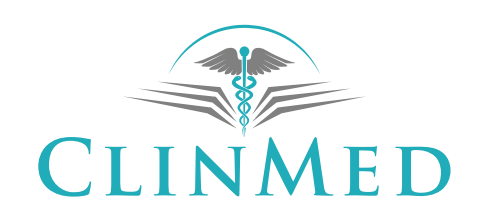

INTERNATIONAL LIBRARY

Citation: Kumar D, Lobo J (2019) Prostatic Ductal Adenocarcinoma: An Unusual Subtype of Prostate Cancer. Int J Oncol Res 2:013

Accepted: May 23, 2019; Published: May 25, 2019

Copyright: (c) 2019 Kumar D, et al. This is an open-access article distributed under the terms of the Creative Commons Attribution License, which permits unrestricted use, distribution, and reproduction in any medium, provided the original author and source are credited. 
expectancy of the patient and the aggressive biological behavior of DAP, he elected to proceed with combined pelvic radiation therapy and androgen deprivation therapy (ADT). During the 1 year follow-up, the patient currently on ADT and remained progression-free with no evidence of local recurrence or distant metastasis.

\section{Discussion}

The differential diagnosis of ductal carcinoma of the prostate includes acinar adenocarcinoma, high-grade prostatic intraepithelial neoplasia, and primary transitional cell carcinoma of the prostate and intraductal carcinoma of the prostate. Although sometimes mixed together, ductal carcinoma of the prostate can be distinguished from acinar adenocarcinoma by the papillary architecture. Similarly, high-grade prostatic intraepithelial neoplasia differs from ductal carcinoma by the glandular morphology without cribriform or sheet patterns [6]. Primary transitional cell carcinoma of the prostate might be confused with ductal carcinoma of the prostate during cystoscopic examination, but histologic examination provides ready distinction. Intraductal carcinoma of the prostate is an atypical glandular lesion that grows into a normal-appearing prostatic duct. Intraductal carcinoma of the prostate is commonly composed of cuboidal epithelial cells with an intact basal cell layer, unlike ductal carcinoma of the prostate [6]. Distinguishing ductal carcinoma is important for clinical management because its presence portends a poorer prognosis and requires aggressive therapy.

The Gleason score is the most widely accepted grading system based on architectural patterns. DAP is assigned a grade of at least $4+4$, due to the aggressive biological behavior and distinctive morphology [7]. Regardless of variable serum PSA levels, samples that exhibit DAP histology normally express PSA and prostatic-specific acid phosphatase in immunostaining; this supports the prostatic origin of DAP and provides evidence for the differentiation of DAP tumors from other lesions. In addition, focal expression of CEA, cytokeratin 7, cytokeratin 20 and caudal type homeobox 2 have been reported in certain cases [5].

Although the clinical course of DAP remains controversial, the majority of studies indicate that the variant of PCa exhibits more aggressive biological behavior, a more advanced stage, a worse 5-year survival rate and a shorter time to progression compared with conventional acinar carcinoma $[3,8]$.

Therapies for DAP include radical prostatectomy, radiation therapy, hormone deprivation, chemotherapy and TURP. Once an initial diagnosis is confirmed using TURP or a transrectal ultrasound-guided needle biopsy, patients are advised to undergo a clinical staging evaluation, including a computed tomography scan of the abdomen and pelvis, bone scan and chest X-ray. If the diagnostic examinations rule out distant metastasis, definitive local therapies, including radical prostatectomies, pelvic lymph node dissections and external beam radiotherapy, may be offered to the patient. Notably, DAP is usually under-staged and appears to be resectable prior to surgery; however, the majority of the patients with DAP have already developed extracapsular invasion. Neoadjuvant approaches prior to surgery are recommended for patients in order to shrink the tumor volume and downstage the DAP. Patients are recommended to receive androgen deprivation following definitive therapies as DAP is prostatic in origin and androgen dependent. In one previous study, radiation therapy was recommended as a more adequate treatment option compared with radical surgery [9].

\section{Conclusion}

DAP is an uncommon finding in prostate biopsies. DAP may be challenging to detect during the early stages of disease due to the lack of distinctive clinical features, and is usually diagnosed at an advanced stage. Definitive therapy (prostatectomy, radiation therapy) is recommended in patients with an established diagnosis of intraductal carcinoma of the prostate on needle biopsy, even in the absence of pathologically documented invasive prostate cancer on needle biopsy, because of the risk of high Gleason grade, high-stage prostatic adenocarcinoma. Knowledge of this tumor is thus important as proper diagnosis and timely intervention can improve patient survival rates.

\section{References}

1. Melicow MM, Patcher MR (1976) Endometrial carcinoma of the prostatic utricle (uterus masculinus). Cancer 20: 17151721.

2. Lee SS (1994) Endometrioid adenocarcinoma of the prostate: A clinicopathologic and immunohistochemical study. J Surg Oncol 55: 235-238.

3. Morgan TM, Welty CJ, Vakar-Lopez F, Lin DW, Wright JL (2010) Ductal adenocarcinoma of the prostate: Increased mortality risk and decreased serum prostate specific antigen. J Urol 184: 2303-2307.

4. Yang XJ, Cheng L, Helpap B, Samaratunga H (2004) Ductal adenocarcinoma. In: Eble JN, Sauter G, Epstein JI, Sesterhenn IA, Pathology and Genetics. Tumours of the Urinary System and Male Genital Organs. ( $3^{\text {rd }}$ edn), IARC, World Health Organization Classification of Tumours, Lyon, 199-201.

5. Epstein JI, Woodruff JM (1986) Adenocarcinoma of the prostate with endometrioid features. A light microscopic and immunohistochemical study of ten cases. Cancer 57 : 111-119.

6. Guo CC, Epstein JI (2006) Intraductal carcinoma of the prostate on needle biopsy: Histologic features and clinical significance. Mod Pathol 19: 1528-1535.

7. Epstein $\mathrm{Jl}$ (2010) An update of the Gleason grading system. J Urol 183: 433-440.

8. Meeks JJ, Zhao LC, Cashy J, Kundu S (2012) Incidence and outcomes of ductal carcinoma of the prostate in the USA: Analysis of data from the Surveillance, Epidemiology, and End Results program. BJU Int 109: 831-834.

9. Orihuela E, Green JM (2008) Ductal prostate cancer: Contemporary management and outcomes. Urol Oncol 26: 368-371. 